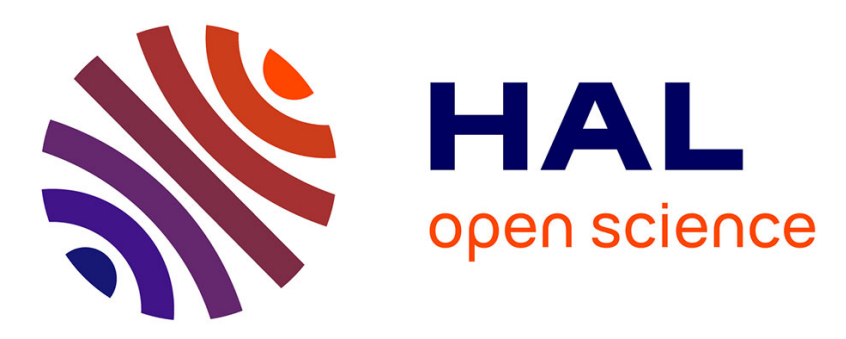

\title{
Les trajectoires mobilitaires : une notion clef pour penser et accompagner les changements de modes de déplacements ?
}

\author{
Laurent Cailly, Marie Huyghe, Nicolas Oppenchaim
}

\section{To cite this version:}

Laurent Cailly, Marie Huyghe, Nicolas Oppenchaim. Les trajectoires mobilitaires : une notion clef pour penser et accompagner les changements de modes de déplacements ?. Flux - Cahiers scientifiques internationaux Réseaux et territoires, 2020, Nº121 (3), pp.52. 10.3917/flux1.121.0052 . hal-03000941

\section{HAL Id: hal-03000941 \\ https://hal.science/hal-03000941}

Submitted on 12 Nov 2020

HAL is a multi-disciplinary open access archive for the deposit and dissemination of scientific research documents, whether they are published or not. The documents may come from teaching and research institutions in France or abroad, or from public or private research centers.
L'archive ouverte pluridisciplinaire HAL, est destinée au dépôt et à la diffusion de documents scientifiques de niveau recherche, publiés ou non, émanant des établissements d'enseignement et de recherche français ou étrangers, des laboratoires publics ou privés. 


\title{
Les trajectoires mobilitaires : une notion clef pour penser et accompagner les changements de modes de déplacements?
}

\author{
Laurent Cailly, Marie Huyghe, Nicolas Oppenchaim
}

Flux, 2020/3 ( $\mathrm{N}^{\circ}$ 121), p. 52-66. DOI : 10.3917/flux1.121.0052. URL : https://www.cairnint.info/revue-flux-2020-3-page-52.htm

Dans un contexte de remise en question de la durabilité des pratiques tout-automobiles (Kaufmann et alii, 2010) et de réflexion sociétale sur les conditions d'une transition vers des mobilités plus durables, cet article cherche à approfondir la compréhension des changements de comportement de mobilité en les replaçant dans le temps long des trajectoires individuelles. Il s'inscrit dans la continuité de travaux antérieurs qui mettent en relation l'évolution des pratiques de mobilité et d'autres composantes des changements biographiques tels les parcours scolaires, professionnels, conjugaux et familiaux des individus (Rocci, 2007 ; Vincent-Geslin, 2010).

Dans cet objectif, en nous inspirant de la notion de trajectoire résidentielle qui réinscrit les choix résidentiels d'un individu dans la totalité de sa trajectoire résidentielle, professionnelle et familiale (Authier, 2010), nous introduisons la notion de trajectoire mobilitaire. Cette notion fait référence aux différentes pratiques de mobilité quotidienne réalisées par un individu au cours de son existence et à la manière dont s'enchaînent et évoluent ces pratiques, envisagées sous l'angle d'un continuum et non comme une série de ruptures sans liens les unes avec les autres. Elle considère que les différentes caractéristiques de la mobilité quotidienne d'un individu forment un système susceptible de se recomposer au cours de l'existence en fonction d'un grand nombre de paramètres et postule que l'évolution de ce système de mobilité au fil du temps obéit à un ordre intelligible dont on peut restituer les logiques et les dynamiques d'évolution, et étudier les régularités. Ce faisant, cette notion permet d'introduire une perspective biographique et longitudinale, moins développée jusqu'alors dans le champ des études des mobilités quotidiennes que dans celui des mobilités résidentielles (Dureau, Imbert, 2014).

Cette mise en perspective des changements de pratiques de mobilité à l'échelle des trajectoires biographiques est au cœur de deux courants de recherche anglo-saxons récents et complémentaires (Cacciari, 2020). Le premier, sous le terme de mobility biographies (Lanzendorf, 2010 ; Müggenburg, Busch-Geertsema, Lanzendorf, 2015 ; Rau, Manton, 2016), considère les pratiques de mobilité comme des activités routinières susceptibles d'évoluer suivant les étapes et les événements-clés (key events) qui marquent le parcours de vie (accès au permis, naissances des enfants, séparation, etc.). Le second courant, qualifié de travel socialization studies (Baslington, 2008), insiste quant à lui plus nettement sur l'influence des expériences de mobilité enfantines sur les changements de pratiques observés à l'âge adulte. La notion de trajectoire mobilitaire que nous développons incorpore différentes avancées théoriques de ces deux courants de recherche; elle permet également de croiser leurs apports respectifs et d'en combler certains angles morts (Aguilera, Cacciari, 2020 ; Cacciari, 2020) : elle vise notamment à considérer avec plus de finesse la pluralité des évolutions 
biographiques et des processus de socialisation qui opèrent dans la transformation des comportements de mobilité.

Cet objectif conditionne en partie les modes de collecte des données (ou en présuppose un renouvellement): alors que les travaux anglo-saxons cités précédemment reposent principalement sur des données quantitatives de panel ou des entretiens centrés sur les moments de changement de pratiques, nos analyses ont été élaborées à partir d'entretiens biographiques approfondis, parfois répétés, et le suivi régulier d'individus en train d'expérimenter de nouveaux modes de déplacement.

Afin d'explorer les contours et les potentialités de la notion de trajectoire mobilitaire, nous nous appuyons sur un travail de représentation graphique schématisant l'évolution des stratégies de mobilité des individus tout au long de leur vie et les différents facteurs à l'origine de leurs changements de pratiques. Cette représentation graphique porte principalement sur les changements de modes de déplacement, objet d'étude des programmes de recherche sur lesquels s'appuie notre article. Néanmoins, la notion de trajectoire mobilitaire, et son opérationnalisation sous une forme graphique, ont vocation à être étendues aux évolutions de l'ensemble des dimensions du système de mobilité, comme par exemple la forme ou l'échelle spatiale des territoires pratiqués.

Nous détaillerons dans un premier temps la notion de trajectoire mobilitaire et les principales hypothèses sur lesquelles elle repose, qui permettent de penser conjointement socialisation $\grave{a}$ la mobilité et dans la mobilité tout au long de la vie. Nous reviendrons ensuite sur les modalités de construction de la représentation graphique de trajectoires mobilitaires, centrée principalement sur les changements modaux. Enfin, dans un troisième temps, nous montrerons que la notion de trajectoire mobilitaire et sa traduction graphique permettent d'enrichir la compréhension des changements de pratiques de mobilité à deux échelles temporelles : d'une part, à l'échelle du processus de changement au cours duquel l'individu passe d'une pratique à une autre, de manière pérenne ou non; d'autre part, à l'échelle de la vie de l'individu, en renseignant notamment les allers-retours entre différents modes de déplacement qui caractérisent un grand nombre d'individus.

\section{Une notion qui articule socialisation à et dans la mobilité}

La notion de trajectoire mobilitaire a été développée à partir d'une réflexion sur les processus de changement de mobilité réalisée dans le cadre du projet «mutualisation et réanalyse de corpus de données sur les mobilités quotidiennes dans les territoires dépendants de l'automobile » (2017-2019) ${ }^{1}$. Nous y avons confronté les résultats de deux recherches : le programme MOUR qui étudie le changement « en train de se faire », en suivant et accompagnant des personnes faisant évoluer leurs habitudes de mobilité (Huyghe, 2015); le programme MODALTER qui s'intéresse de manière rétrospective aux tests d'alternatives à la voiture, en les replaçant dans un parcours global grâce à des entretiens biographiques (Oppenchaim, Fouquet, Pourtau, 2017). Nous avons ensuite réanalysé la soixantaine d'entretiens menés au cours de ces deux recherches avec des habitants de l'espace rural et périurbain tourangeau présentant une réelle diversité en termes sociodémographiques, de contexte d'habitat ou d'usages des modes. Cette réanalyse a été nourrie par la découverte des

1 Cette recherche collective financée par le Forum Vies Mobiles, avait pour objectif de réinvestir six recherches récentes, menées entre 2009 et 2016 à l'UMR 7324 CNRS-CITERES, en les ré-analysant à partir de trois thématiques transversales : les formes de la mobilité quotidienne, le vécu du temps de déplacement et les processus de changement modaux. 
travaux de deux courants de recherche anglo-saxons, les mobility biographies $-M B-$ et les travel socialization studies - TSS. Développés au début des années 2000 (Lanzendorf, 2010), ces deux courants s'intéressent au temps long des pratiques mobilitaires et s'attachent à faire des liens entre les pratiques mobilitaires et divers éléments biographiques ou étapes-clés en matière de mobilité qui surviennent au cours de la vie (Cacciari, 2020). Ils offrent ainsi des pistes pour penser conjointement socialisation à la mobilité et dans la mobilité (Cacciari, 2020), en soulignant, d'un côté, l'influence des expériences de mobilité vécues dans l'enfance (TSS) et, de l'autre (MB), l'importance de certaines étapes et événements du parcours de vie sur l'évolution des pratiques de mobilité des individus. Ces événements peuvent être directement liés à la mobilité (comme l'obtention du permis, la panne d'un véhicule, etc.) ou concerner d'autres sphères d'activités comme la mise en couple, un déménagement, etc. (Rau, Manton, 2016) : si les dispositions vis-à-vis de la mobilité, incorporées durant la socialisation primaire puis secondaire, influencent les changements modaux, elles peuvent également évoluer lorsque les individus expérimentent de nouveaux moyens de déplacement à la suite d'événements-clés déstabilisant les routines habituelles de déplacement, notamment lorsque ces événements biographiques modifient la géographie de l'habiter (changement de lieu de résidence, de travail, de scolarisation des enfants).

Ce processus de mutualisation et de réanalyse des données nous a conduit à formuler trois hypothèses centrales qui permettent, selon nous, de mieux comprendre les processus de changement de mobilité tout au long de la vie.

La première hypothèse, relativement classique parmi les chercheurs travaillant sur les bifurcations biographiques (Grossetti, 2006), repose sur l'idée que le déclenchement et le déroulement des processus de changement s'expliquent à la fois par des déterminations à long terme, propres à l'individu et aux contextes dans lesquels il évolue, mais aussi par des événements pouvant déstabiliser les routines individuelles. Nos matériaux permettent ainsi d'identifier quatre grandes familles de facteurs pouvant rendre intelligibles l'engagement d'un individu dans un processus de changement modal et le déroulé de celui-ci :

- tout d'abord, les ressources, valeurs, compétences et dispositions acquises par l'individu au cours de son existence dans différentes sphères de socialisation. Ces différents éléments qui participent de la «motilité » (Kaufmann, 2014) des individus sont acquis à la fois dans l'enfance, en particulier dans la famille, mais aussi à l'âge adulte. Sur ce point, nous nous distinguons du courant des travel sozialisation studies qui accorde une place décisive à la socialisation enfantine et néglige plus largement la dimension d'apprentissages dans la pratique et l'émergence au cours de la vie de schèmes d'action dissonants (Cailly, 2007; Oppenchaim, 2016; Cacciari, 2020). L'individu découvre ainsi des ressources, acquiert des compétences, incorpore des dispositions lorsque lui, ou un autre membre de son ménage, modifie son système de mobilité ;

- l'évolution des contextes (sociaux, familiaux, professionnels ou géographiques) qui caractérisent son parcours biographique ; chaque moment biographique peut mettre à l'épreuve le système de mobilité ; il redéfinit la carte des ressources, des contraintes et plus largement des paramètres qui participent à faire évoluer les choix de mobilité ;

- les éléments fortuits ou contingents : des opportunités ou des perturbations extérieures qui surviennent au cours de l'expérience et impactent de manière décisive les 
pratiques de mobilité d'un individu, tels qu'une grève ferroviaire, l'arrivée d'un nouveau collègue faisant du covoiturage, les changements d'horaires professionnels du conjoint, etc. ;

- enfin, le vécu de la mobilité, en particulier de l'espace et du temps de déplacement, et les représentations associées par l'individu aux différents modes et déplacements sont à considérer. L'habitabilité ${ }^{2}$ du mode utilisé, la pénibilité ressentie ou encore la découverte d'usages potentiels contribuent à la stabilisation ou à la révocation des routines de mobilité.

La deuxième hypothèse est d'envisager les pratiques de mobilité individuelles comme une succession de processus d'expérimentation au cours desquels les individus peuvent passer par des phases de préparation, d'action, de réflexivité, pour aboutir à la pérennisation de nouvelles routines ou, au contraire, à un retour aux routines antérieures. Nous nous appuyons pour cela assez librement sur le modèle de psychologie sociale de Prochaska et Di Clemente (1982) (voir Figure 1), en identifiant des phases successives par lesquelles peut passer un individu qui fait évoluer ses pratiques, notamment une phase de pré-contemplation, pendant laquelle l'individu est globalement satisfait de ses pratiques et ne cherche pas à les modifier ; une phase de contemplation, durant laquelle l'individu ressent une insatisfaction légère à importante vis-à-vis de ses pratiques et s'ouvre au changement ; une phase de détermination, caractérisée par la prise de renseignements et une réflexion autour du changement de mode envisagé et de ses implications ; une phase d'action où l'individu expérimente de nouvelles pratiques ; enfin, une phase de maintien qui se caractérise par la stabilisation à plus ou moins long terme du nouveau comportement de mobilité. Ce modèle permet notamment de mieux situer l'état d'esprit d'un individu lorsqu'il s'engage dans l'expérimentation d'un nouveau mode de déplacement : lorsqu'il est confronté à un événement extérieur, par exemple, une panne de voiture, un individu peut être «totalement fermé au changement » (on le situera en phase de pré-contemplation) ou « ouvert au changement mais pas prêt à agir » (on le situera en phase de contemplation), ce qui n'est pas sans influence sur la suite du processus. Ce

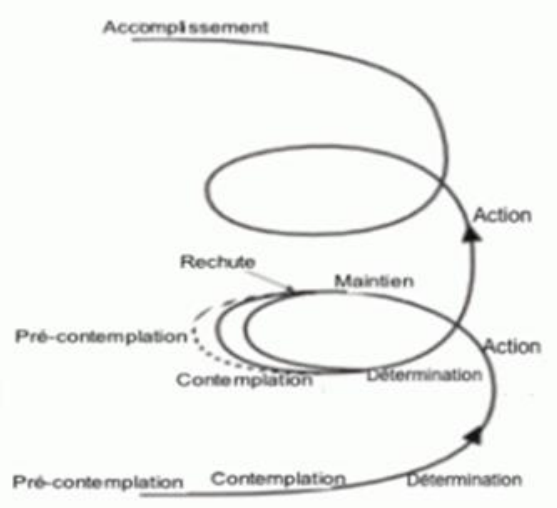
modèle permet également, en discernant des étapes, de mieux comprendre la dynamique très individualisée du changement, tantôt marquée par un enchaînement progressif des phases, tantôt par le saut d'une ou de plusieurs étapes, notamment lorsque le changement est contraint (par la panne d'un véhicule, par une longue grève, etc.), tantôt par un retour avant la fin de l'expérimentation à l'ancien mode, qui, parfois, pose néanmoins les bases de changements ultérieurs.

2 Le terme d'habitabilité ne désigne pas seulement un ensemble de qualités et de propriétés intrinsèques de l'espace géographique (ici les espaces fréquentés lors du déplacement : habitacle automobile, gare, wagon de train, piste cyclable, etc.), mais également (et surtout) un ensemble de compétences et d'arts de faire mis en œuvre par l'individu pour réduire les contraintes et maximiser les ressources que livre l'espace géographique au cours de ses activités quotidiennes. 
Cela nous conduit à formuler une troisième hypothèse, celle de l'absence de linéarité des processus de changements (Oppenchaim, Fouquet, Pourtau, 2017) qui nous invite à rompre avec la conception du changement de pratiques comme un moment de rupture, sous l'effet d'événements-clés, entre deux routines de déplacements. Cette conception, dominante dans le courant des mobility biographies (Mattioli, 2019), est particulièrement forte dans les politiques publiques visant à réduire la part modale de la voiture. Elle survalorise le changement, le considérant comme un moment clé avec un «avant» et un «après », comme une conversion radicale d'un mode vers un autre: il existerait des pratiques antérieures (généralement, des pratiques automobiles) et des pratiques postérieures au changement (généralement, altermobiles). Nos matériaux montrent que cette conception du changement comme «moment fatidique » (Javeau, 2006) n'est guère fidèle aux pratiques de nos enquêtés

- elle donne l'impression qu'une fois le changement effectué, les nouvelles pratiques sont adoptées une fois pour toutes et deviennent immuables : on observe en réalité que les individus font évoluer leurs pratiques mobilitaires tout au long de leur vie, au gré de leurs diverses contraintes, opportunités, préférences, envies, etc. ;

- elle ne reflète pas non plus la complexité des routines des individus, qui font fréquemment des allers-retours entre les différents modes ou les utilisent conjointement durant une même période, valorisant ainsi un répertoire d'expériences et de compétences ;

- elle oublie que le changement ne se fait pas instantanément mais est un processus, potentiellement long, qui permet de faire évoluer des habitudes, des routines, vers de nouvelles pratiques ;

- enfin, cette conception du changement ne permet pas de prendre en compte l'histoire de l'individu, ses expériences, ses réussites et ses échecs: le processus d'expérimentation d'alternatives à la voiture constitue rarement une rupture soudaine dans la trajectoire des individus, qui entrerait en contradiction avec leur socialisation antérieure à la mobilité.

La notion de trajectoire mobilitaire que nous proposons permet d'appréhender et de traiter conjointement ces trois hypothèses, afin de mieux comprendre la logique des évolutions des pratiques de mobilité quotidienne au cours de la vie et le rôle qu'y joue la socialisation à la mobilité et dans la mobilité :

La notion de trajectoire mobilitaire s'intègre dans une démarche générale visant à mieux comprendre, à travers le prisme des mobilités, la construction du rapport des individus à l'espace et la diversité des modes d'habiter. Elle vise à décrire et expliquer l'évolution des pratiques de déplacement d'un individu au cours de son existence. Elle considère que les différentes caractéristiques de la mobilité (distances parcourues, vitesses, temporalités, modes, échelles, motifs, lieux de destination, parcours et habitabilité du déplacement) forment un système susceptible de se recomposer au cours de l'existence en fonction d'un grand nombre de paramètres. Ainsi, le système de mobilité qui caractérise un individu à un instant $T$ fait sens et s'explique à la lumière du temps long, notamment en fonction des matrices de socialisation, des expérimentions passées ou encore de l'évolution des contextes (sociaux, familiaux, professionnels ou géographiques) qui caractérisent le parcours biographique. 
Suivant cette conception, l'évolution des pratiques de mobilité - que synthétise la trajectoire mobilitaire - obéit à un ordre intelligible dont on peut restituer les logiques et les dynamiques d'évolution et étudier les régularités. Celles-ci relèvent de nombreux déterminants, des facteurs structurels aux logiques d'acteurs, des dispositions incorporées aux effets contextuels. La socialisation primaire ou secondaire par exemple, l'évolution dans le cycle de vie (effet de la parentalité et de l'accompagnement des enfants), la dynamique des choix résidentiels ou encore de l'évolution des localisations et des besoins de mobilité à titre professionnel participent à la définition de la trajectoire mobilitaire. La notion se donne donc pour ambition de mettre l'accent sur les conditions structurelles tramées au long court comme sur les logiques d'opportunité et contextuelles inscrites dans le temps court, qui contribuent aussi à modifier les logiques d'action. Elle accorde une place déterminante au rôle de l'expérimentation, de l'acquisition de compétences et de la réflexivité dans l'analyse des changements de comportement de mobilité.

\section{La représentation graphique de la notion de trajectoire mobilitaire}

Nous avons voulu opérationnaliser la notion de trajectoire mobilitaire sous la forme d'une représentation graphique permettant de visualiser d'une part les évolutions du système de mobilité d'un individu, d'autre part les différents facteurs, de court et de long terme, expliquant ces évolutions. Dans cette représentation graphique, l'évolution du système de mobilité est renseignée par les changements modaux de l'individu. Des représentations complémentaires, centrées sur des évolutions d'autres dimensions du système de mobilité (taille de l'espace pratiqué par exemple), permettront dans le futur, nous l'espérons, d'approfondir les potentialités de la notion de trajectoire mobilitaire.

Nous avons testé la pertinence de cette représentation graphique à partir de notre matériau d'enquête. Les entretiens réalisés n'ayant pas tous pour ambition initiale de retracer les parcours mobilitaires des individus, certaines trajectoires reconstituées sont plus lacunaires que d'autres, notamment en ce qui concerne les pratiques enfantines des individus et leur socialisation aux différents modes. Ainsi, selon la richesse des entretiens, certaines trajectoires peuvent être reconstruites en prenant en compte la totalité de la vie de l'individu, depuis sa naissance ou son enfance; d'autres ne permettent de prendre en compte que les quelques dernières années de l'individu interrogé. Cette situation, classique dans la littérature (Rau, Manton, 2016), limite la possibilité de comparer exhaustivement les différentes trajectoires ou de comprendre, pour certains enquêtés, la manière dont la socialisation secondaire à la mobilité s'articule avec les dispositions héritées de la socialisation primaire par exemple.

\section{La construction de la représentation graphique}

La représentation graphique que nous proposons présente, sur un même schéma (Figure 2), l'ensemble des éléments participant à la compréhension de l'évolution des pratiques de mobilité de l'individu tout au long de sa vie.

Elle s'articule autour de deux axes :

- un axe chronologique vertical, permettant de retracer la vie de l'individu ; 
- un axe horizontal comprenant une colonne principale, située à gauche, renseignant les différents modes de transport utilisés par l'individu au cours de sa vie ; et six colonnes renseignant les familles de facteurs permettant de comprendre l'évolution des pratiques de mobilité de l'individu présentées dans la partie précédente : le vécu de la mobilité et les représentations associées aux modes ; les valeurs, ressources et dispositions de l'individu; les événements extérieurs impactant ses pratiques; l'évolution de sa situation professionnelle, résidentielle et familiale (avec un fond coloré, afin de différencier ces trois dernières colonnes, car les informations présentes ne font pas directement référence aux pratiques modales des enquêtés, contrairement à ce qu'on retrouve dans les colonnes précédentes).

Cette grille est complétée par une première couche d'information, constituée de l'ensemble des éléments évoqués au cours de l'entretien par l'enquêté au sujet de sa mobilité et de celle des autres membres de son ménage. Ces éléments reposent sur une co-construction entre l'individu et le chercheur qui l'interroge durant l'entretien. Ils sont synthétisés sous forme de courts textes qui sont rangés dans les sept colonnes du graphique, de manière chronologique à partir de l'axe vertical. Ce rangement chronologique se fait soit au regard d'indications données explicitement par l'enquêté, soit par déduction faite par le chercheur à partir du récit que fait l'individu de sa trajectoire.

Cette première couche d'information est ensuite complétée par une seconde couche, que nous appelons « le fil interprétatif ». Ce fil relie l'ensemble des éléments que le chercheur juge centraux pour décrire et rendre compte des logiques qui structurent la trajectoire mobilitaire de l'individu. Ce fil relie bien entendu les éléments que les enquêtés mettent en avant dans leur récit pour justifier leur changement de pratique. Il intègre aussi des éléments que le chercheur identifie comme structurants, sans que l'individu ne les ait mis en avant et validés $a$ posteriori. À l'inverse, certains éléments évoqués par l'enquêté au sujet de sa mobilité peuvent être écartés, si le chercheur et l'enquêté jugent qu'ils n'ont guère d'influence sur l'évolution des pratiques de l'individu.

Ce fil permet également de représenter les différents états psychologiques de l'individu vis-àvis de ses pratiques de mobilité au fil de sa trajectoire en reprenant les phases du modèle de Prochaska et Di Clemente (1982), identifiées par un code couleur spécifique. Cette représentation des phases par lesquelles passe un individu « en changement » par l'intermédiaire du fil interprétatif est beaucoup plus adaptée à la notion de trajectoire mobilitaire que la représentation sous forme de spirales proposée par ces deux derniers auteurs (Figure 1). Cette dernière a été pensée dans le cadre d'opérations visant à sevrer l'individu de pratiques addictives, en particulier de tabagisme, ce qui explique à la fois le vocabulaire employé ( « rechute ») et la forme en spirale marquant le retour à une pratique addictive. Au contraire, le fil interprétatif représente le caractère dynamique des trajectoires mobilitaires, faites d'expérimentations, d'essais-erreurs, d'acquisition de nouvelles compétences, de développement de nouvelles valeurs ou convictions, etc., se traduisant, ou non, par une évolution dans les modes de déplacement pratiqués. La trajectoire de Mme Hiver présentée ci-dessous (Figure 2) permet de montrer ce que nous intégrons dans la trajectoire mobilitaire et la façon dont nous pouvons tisser des liens entre les éléments. 
Figure 2. La représentation graphique de la trajectoire de Mme Hiver

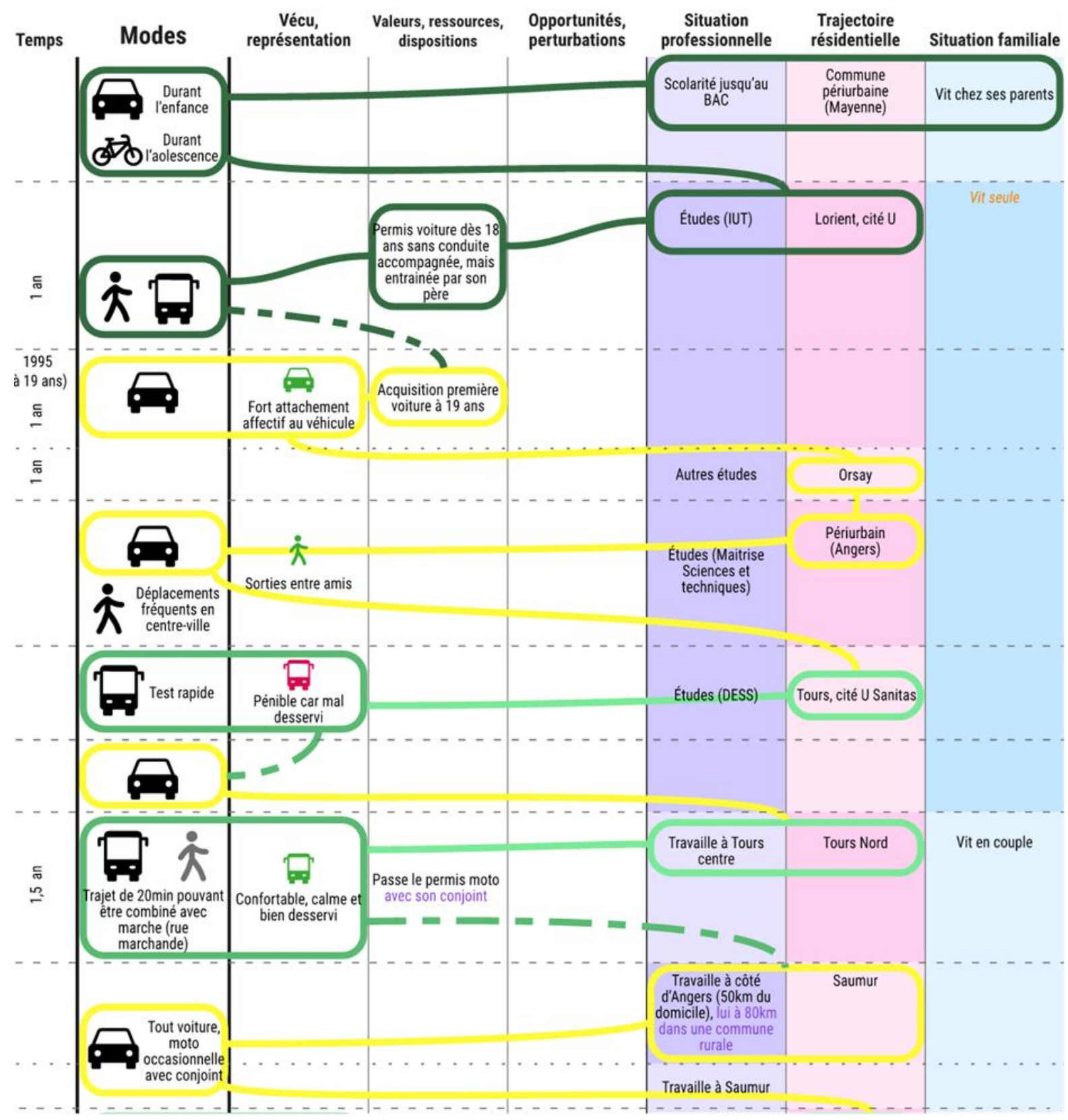




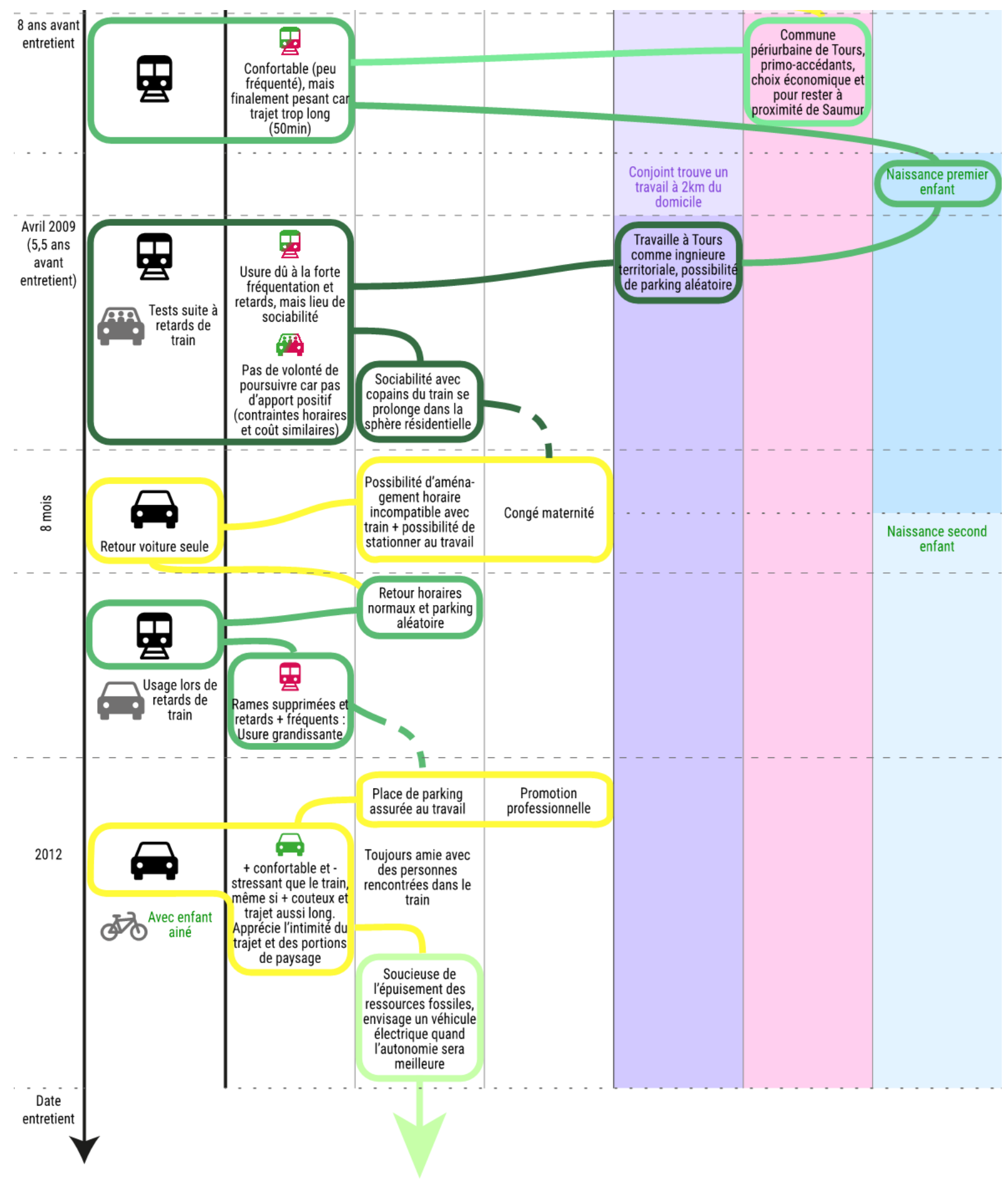




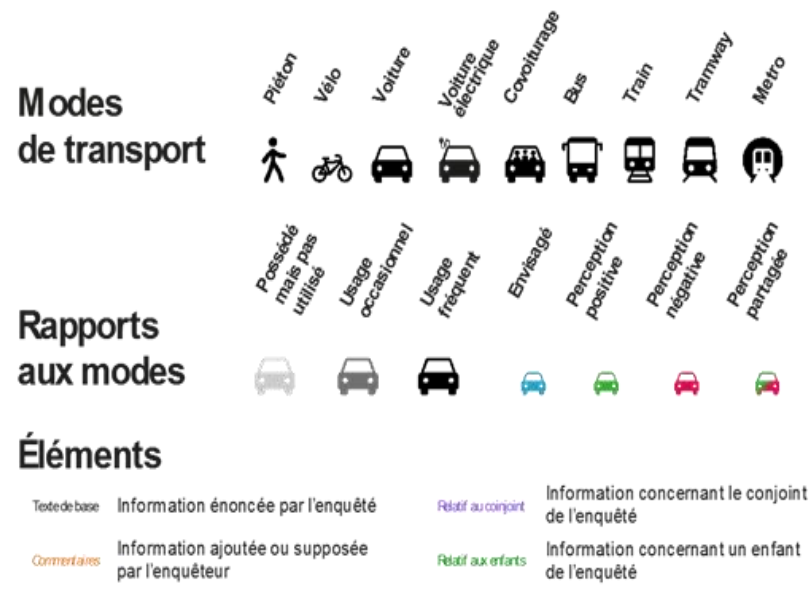

\section{Délimitations temporelles}

Périodes « principales », marquant un changement dans les modes de déplacement

Périodes secondaires marquant d'autres types de changements

\section{Fil interprétatif, phases de changement}

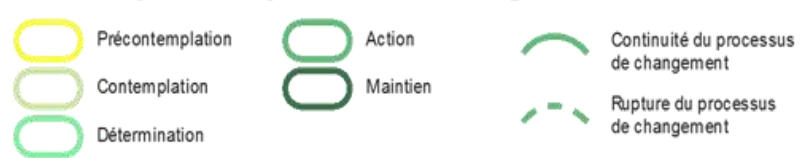

La trajectoire mobilitaire de Madame Hiver retrace l'évolution de ses pratiques de mobilité depuis son enfance. Employée à la mairie de Tours et résidant en lointain périurbain, celle-ci se rend sur son lieu de travail, situé à quelques minutes à pied de la gare, en TER. Au fil des années, elle a développé des relations de sociabilité avec deux navetteuses, rencontrées sur le quai de la gare, qui elles aussi travaillent en hyper-centre, à proximité de son lieu d'emploi. Elles ont pris l'habitude de se retrouver sur leur pause méridienne et se fréquentent également en dehors de leur travail, dans leur commune de résidence. Ces contreparties sociales et amicales n'estompent pas pour autant l'usure de l'usage des transports en commun qui s'est progressivement installée chez Mme Hiver. Elle mentionne par ailleurs les grèves répétées et la présence en nombre de lycéens bruyants comme facteurs explicatifs de sa lassitude. Lors de sa première grossesse, elle a obtenu une place de stationnement dans le parking de la mairie et abandonné le train pour la voiture. Ce changement ne lui fait pas gagner du temps mais du confort de déplacement. À l'issue de son congé maternité, elle a dû rendre cette place et reprendre le train, à regret. Il y a quelques années, suite à une promotion professionnelle, elle s'est vue attribuer une place de stationnement, définitive celle-ci. Depuis, elle organise ses trajets domicile-travail exclusivement en voiture individuelle.

\section{Qu'apporte la représentation graphique?}

Représenter graphiquement une trajectoire mobilitaire présente différents intérêts.

La représentation graphique est d'abord un outil d'aide à l'analyse, permettant de schématiser les processus de changement modaux, à partir de l'information épaisse contenue dans les entretiens. La construction de la trajectoire s'apparente alors à une forme particulière d'analyse thématique et de codage de l'entretien, au sens où elle identifie, différencie et donne sens aux données extraites, et en propose une interprétation. Cette représentation permet de reconstituer la logique des trajectoires et de pouvoir les comparer rapidement entre elles, d'en extraire des singularités et des différences, sans avoir nécessairement besoin de se replonger 
dans l'ensemble du corpus d'entretiens. Elle est ainsi particulièrement adaptée dans le cadre des recherches collectives portant sur un nombre conséquent d'enquêtés. Cet outil permet notamment de comparer l'enchaînement des étapes et des phases traversées, et donc d'établir des parcours types, à l'image des travaux sur les trajectoires résidentielles qui montrent que celles-ci suivent une certaine logique et sont susceptibles de s'enchaîner selon un ordre intelligible (Authier, 2010). Sur de petits échantillons qualitatifs, l'outil des trajectoires mobilitaires fournit sur ce point une alternative aux méthodes d'appariement optimal dont la finalité est de bâtir une typologie de séquences (Lesnard, Saint-Pol, 2006). Ces comparaisons permettent à terme de dégager des éléments explicatifs des processus de changement de mobilité.

La représentation graphique est aussi un outil de visualisation des trajectoires des individus, permettant au chercheur d'exemplifier ses analyses à partir des cas étudiés, et au lecteur d'accéder rapidement à la logique des trajectoires en complément des explications du chercheur. Le lecteur dispose ainsi d'une vue globale de la trajectoire mobilitaire d'un individu (ses expériences modales, les compétences qu'il a développées, les ressources qu'il a découvertes, etc.) qui lui permet de contrôler l'analyse et l'interprétation qu'en fait le chercheur, voire d'y trouver des éléments explicatifs qui auraient échappé à ce dernier. Ainsi, la représentation graphique ci-dessus permet en quelques minutes de prendre connaissance de la trajectoire de Mme Hiver, d'une manière bien plus complète qu'avec une synthèse du chercheur et bien plus rapide qu'avec la lecture de son entretien biographique. Pour autant, la représentation graphique n'a pas pour vocation à se substituer à l'analyse et au texte argumentatif du chercheur. Issu d'une première analyse, cet outil fournit une interprétation synthétique et intermédiaire qui rend compte d'un ensemble de logiques et appelle, en retour, approfondissements et développements. C'est en définitive dans le corps du texte argumentatif que le chercheur explicite et développe les logiques qui participent à la transformation des systèmes de mobilité.

\section{Saisir le temps court et le temps long des processus de changement}

Ces différentes vertus de la notion de trajectoire mobilitaire et de sa représentation graphique permettent de mieux comprendre et expliquer les processus de changement de pratiques de mobilité, sur le temps court du test d'un nouveau mode de déplacement, mais aussi à l'échelle de la trajectoire biographique des individus. Afin d'exemplifier cette articulation entre les différentes temporalités du changement que permettent de voir la notion de trajectoire mobilitaire et sa représentation, nous allons nous intéresser à un point particulier : la manière dont les individus incorporent à différents moments de leur vie des compétences et des dispositions vis-à-vis de la mobilité, potentiellement dissonantes, et comment ces éléments variés de socialisation permettent de rendre intelligible, en complément d'autres facteurs, la logique des trajectoires.

\section{Sur le temps long : l'importance relative de la socialisation enfantine}

La représentation de la trajectoire de Mme Hiver présentée ci-dessus illustre tout d'abord l'intérêt que revêt la notion de trajectoire mobilitaire pour saisir la complexité des pratiques et des changements modaux. Elle permet de mettre en évidence les allers-retours entre différents modes (ici entre la voiture et le train principalement) et d'interroger les facteurs qui en sont à l'origine. La socialisation à la voiture, l'évolution des contextes de résidence et de travail et les logiques d'opportunité expliquent, dans son cas, la réversibilité à double sens des pratiques 
mobilitaires. Les allers-retours entre les modes étant fréquents, la trajectoire de Mme Hiver est particulièrement exemplaire. Beaucoup évoquent en effet des évolutions, plus ou moins marquées, de leurs pratiques de mobilité au cours de leur vie, y compris les utilisateurs réguliers et satisfaits de la voiture ${ }^{3}$. Ce constat modifie la représentation des territoires dits « dépendants de l'automobile ». Ainsi, bien que très attachés à la voiture (Cailly, 2008), ces habitants du périurbain et du rural ont engagé, pour une part significative d'entre eux, une réflexion autour d'alternatives modales et se livrent à des formes d'expérimentations ordinaires (Dodier, 2015), comme l'illustre l'exemple de Mme Hiver lorsqu'elle teste le covoiturage. Ils se tiennent à l'écoute d'expériences tentées par certains collègues, testent le covoiturage ou d'autres modes, en particulier lors d'une grève (comme dans le cas évoqué cidessus) ou d'une indisponibilité temporaire de leur véhicule. Si ces réflexions et expérimentations n'entraînent pas un abandon de la voiture individuelle, elles montrent en miroir que le choix automobile est rarement fondé sur un rejet des autres modes, notamment des transports collectifs qui impliquent le côtoiement d'autres citadins (Lévy, 2003). Plus largement, il ressort de notre recherche que de nombreux enquêtés se caractérisent par des allers-retours ou des combinaisons entre différents modes, notamment entre voiture et altermobilité, sur des périodes plus ou moins longues, y compris des personnes témoignant d'un fort attachement à l'automobile.

La trajectoire de Mme Hiver présente également un autre intérêt : elle permet de montrer l'impact des pratiques enfantines ou adolescentes sur les pratiques adoptées ou expérimentées à l'âge adulte. Ainsi, en comparant les trajectoires mobilitaires de nos enquêtés les plus attachés à l'automobile, nous constatons quasi-systématiquement une enfance dans le périurbain ou dans le rural, marquée par un usage important de la voiture. Plus largement, la comparaison des trajectoires mobilitaires permet de confirmer l'existence d'une mémoire modale (Vincent-Geslin, 2010), qui amène des individus socialisés à des modes autres que la voiture à tester ensuite plus facilement des alternatives à la voiture, voire à les adopter de manière pérenne. Elle nuance néanmoins l'impact de cette mémoire modale sur deux points :

- d'une part, celle-ci serait surtout opérante lorsque la première socialisation au mode a été une expérience positive, associée à un ressenti agréable. Dans le cas où le mode est associé à des souvenirs négatifs (parce que l'expérience a été désagréable, réalisée dans un contexte peu apprécié par l'individu par exemple), la mémoire modale conduit au contraire les individus à refuser de les emprunter, voire à ne pas/plus les considérer comme une alternative possible ;

- d'autre part, les effets de la socialisation enfantine à la mobilité ne sont pas mécaniques, ils exercent une force de rappel sous la forme de répertoires d'action disponibles dans certains contextes, mais ils n'entraînent pas automatiquement l'adoption à l'âge adulte du mode utilisé durant sa jeunesse. En effet, comme le montre l'exemple de Mme Hiver, la trajectoire résidentielle et professionnelle des individus les conduit bien souvent à expérimenter d'autres modes de déplacement sur des périodes plus ou moins longues, durant lesquelles ils peuvent acquérir de nouvelles dispositions vis-à-vis de la mobilité.

Sur le temps court : la découverte de ressources et de compétences durant le changement

\footnotetext{
3 En l'absence de contraintes s'opposant à son utilisation (coût économique de la mobilité trop élevé, difficultés de stationnement, travaux), ceux-ci se déplacent, pendant la majorité de leur vie, en voiture.
} 
La comparaison des trajectoires mobilitaires de nos enquêtés permet aussi de mieux comprendre le déroulement du processus d'expérimentation de nouveaux modes de déplacement. Elle montre que deux types de changement semblent influencer le déroulement du processus : les changements libres, spontanés, souhaités ; les changements sous contrainte (en raison d'un événement extérieur ou d'une rupture biographique par exemple).

Cette diversité de «degrés de motivation » au changement influence le déroulement du processus de changement, en particulier le nombre et l'enchaînement des phases identifiées dans le modèle de Prochaska et Di Clemente (1982) : si on retrouve le schéma-type de ce modèle (i.e. un passage par chacune des six étapes du changement) chez les individus qui souhaitent faire évoluer leurs pratiques, ceux qui sont contraints au changement ne passent pas par toutes les phases, comme le montre ci-dessous l'exemple de Mme Da Silva (Figure 3) qui, confrontée à la panne de son véhicule, passe immédiatement à une phase d'action. L'origine du processus influence également les temporalités du changement ou la manière dont les individus vont percevoir l'abandon éventuel du nouveau mode testé et le retour à l'ancien mode, le plus souvent la voiture individuelle : un changement souhaité et volontaire pourra notamment se traduire par une répétition des tentatives de changement, y compris en cas d'expérimentations non satisfaisantes.

La comparaison des trajectoires de notre corpus permet d'approfondir de manière plus générale la question des facteurs de déclenchement du changement. La littérature (notamment Rau, Manton, 2016 ; Greene, Rau, 2016 ; Schoenduwe et alii, 2015) identifie trois familles de facteurs, liés à l'individu ou à son environnement, qui peuvent déstabiliser les routines et mener à une évolution des pratiques :

- une insatisfaction vis-à-vis des pratiques: fatigue, pénibilité ou inconfort des déplacements; budget économique ou temporel de la mobilité trop élevé ; tension/décalage entre valeurs (environnementales notamment) et pratiques; émergence de nouvelles appétences ou désirs de mobilité, etc. ;

- des opportunités : création d'une nouvelle offre de transport ; apparition/suppression de stationnements; rencontre avec une offre via un proche (qui propose de covoiturer ou d'utiliser un mode alternatif); opportunité de vendre sa voiture (Deleuil, Barbey, Sintès, 2017) ; proposition de participer à une opération de marketing individualisé ;

- des perturbations des habitudes : micro-événement (grèves de transport, travaux, accidents, retards répétitifs, changement d'horaires des transports, panne de voiture) ou rupture biographique (naissance, décès, séparation, déménagement, changement de lieu de travail).

L'analyse de notre corpus permet de montrer que ces facteurs ne vont pas uniquement dans le sens de l'adoption de pratiques altermobiles : certains peuvent au contraire déstabiliser des pratiques altermobiles et conduire l'individu à (re)prendre la voiture. Les trajectoires de nos enquêtés montrent également que l'impact de ces différents facteurs diffère selon les caractéristiques sociodémographiques (revenu, localisation résidentielle, etc.) de l'individu qui y est soumis, mais également selon ses valeurs, compétences, normes, représentations (Huyghe, 2015). Ainsi, une panne de voiture peut induire un changement si elle s'impose à un individu sans possibilité de racheter un véhicule ou disposant d'alternative; sinon, elle pourrait n'induire qu'un rachat d'une voiture, mais pas un changement de pratiques de 
mobilité. De même, la naissance d'un enfant est une rupture biographique qui perturbe les habitudes de mobilité : chez certains individus enquêtés elle induit un retour à la voiture, chez d'autres non. L'analyse des trajectoires mobilitaires permet alors de monter que, pour un certain nombre d'enquêtés, c'est l'articulation de plusieurs de ces facteurs (qui peuvent survenir à différents moments, voire à plusieurs années d'intervalle) qui sont nécessaires pour déclencher un processus de changement. Par exemple, l'argument du confort ou de la fatigue du déplacement ne suffit pas à enclencher un changement... jusqu'à ce qu'un autre argument financier vienne s'ajouter, qui rend la pratique intenable.

Enfin, les trajectoires de nos enquêtés montrent l'importance des ressources découvertes tout au long du processus d'expérimentation de nouveaux modes de déplacement, qui viennent consolider (ou au contraire ébranler) l'usage pérenne de ces nouveaux modes. Ces ressources peuvent être externes à l'individu, matérielles (douches, stationnement vélos, offres de mobilité jusqu'alors inconnues, etc.) ou humaines (compagnons de train qui peuvent devenir covoitureurs en cas de grève, collègues qui peuvent prêter une voiture sur le lieu de travail, etc.). Elles peuvent également prendre la forme de compétences de mobilité qui se développent ou réapparaissent (compétences développées pendant l'enfance ou l'adolescence, qui n'étaient plus valorisées). Ces ressources sont souvent insoupçonnées, voire insoupçonnables avant le début du processus du changement, et difficiles à anticiper malgré une phase de préparation. Ainsi, Mme Da Silva, auxiliaire de puériculture âgée de 38 ans, résidant dans le périurbain tourangeau et travaillant dans un quartier du centre de Tours situé à quinze minutes à pied de la gare, qui a testé le train après une panne de voiture, a peu à peu découvert différentes ressources qui ont participé à consolider son expérience. Elle a par exemple appris l'existence des SMS envoyés par l'exploitant du réseau ferroviaire régional en cas de perturbation de l'offre, qui « lui permettent de s'organiser, de savoir si elle prend sa voiture ». La possibilité d'anticiper qu'offrent les SMS est une sécurité supplémentaire qui lui permet de continuer à prendre le train, malgré les perturbations fréquentes sur sa ligne. De même, elle a découvert au fur et à mesure de son expérimentation diverses ressources apportées par ses trajets en train : la possibilité de profiter du déplacement pour retrouver le temps et le goût de la lecture; le développement de relations de sociabilité avec d'autres parents de sa commune faisant le même trajet; un rééquilibrage de l'exercice des tâches ménagères à l'intérieur de son ménage, car elle part plus tôt de son domicile.

La représentation graphique de sa trajectoire permet ainsi de montrer comment ses nombreux changements de modes de déplacement sont influencés par les quatre familles de facteurs identifiées dans la première partie : sa trajectoire résidentielle, avec notamment le passage d'un habitat au centre de Tours au périurbain ; des dispositions favorables à l'utilisation de la marche et des transports en commun depuis sa jeunesse dans une ville moyenne du Brésil ; le vécu des temps de déplacement qui lui font préférer le train à la voiture ; des perturbations, tels que les vols de ses vélos, la panne de sa voiture ou bien encore les changements d'horaires professionnels de son mari qui la contraignent à abandonner à regret le train.

Figure 3. La représentation graphique de la trajectoire de Mme Da Silva 


\section{Mme Da Silva - Modalter}

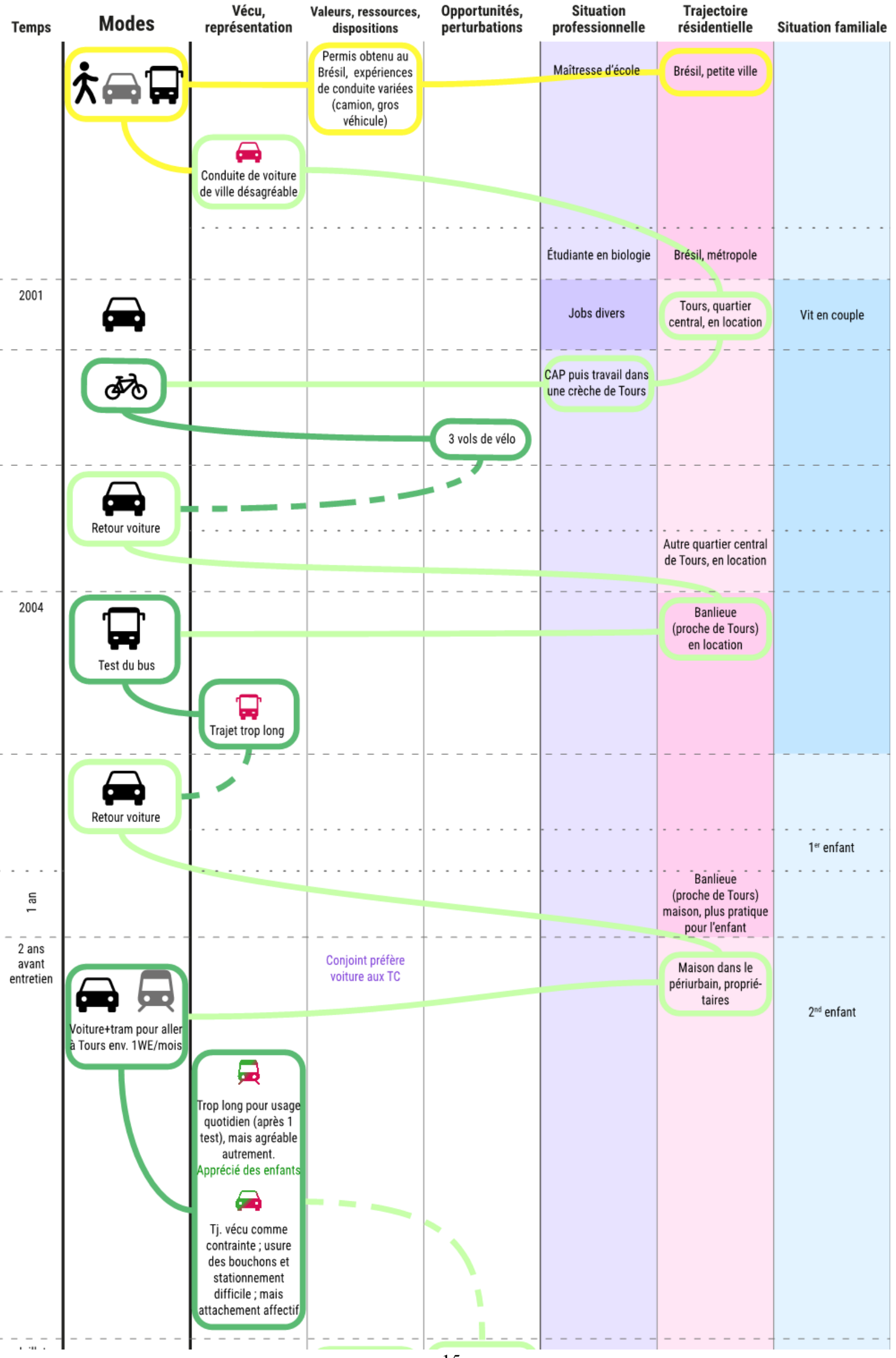




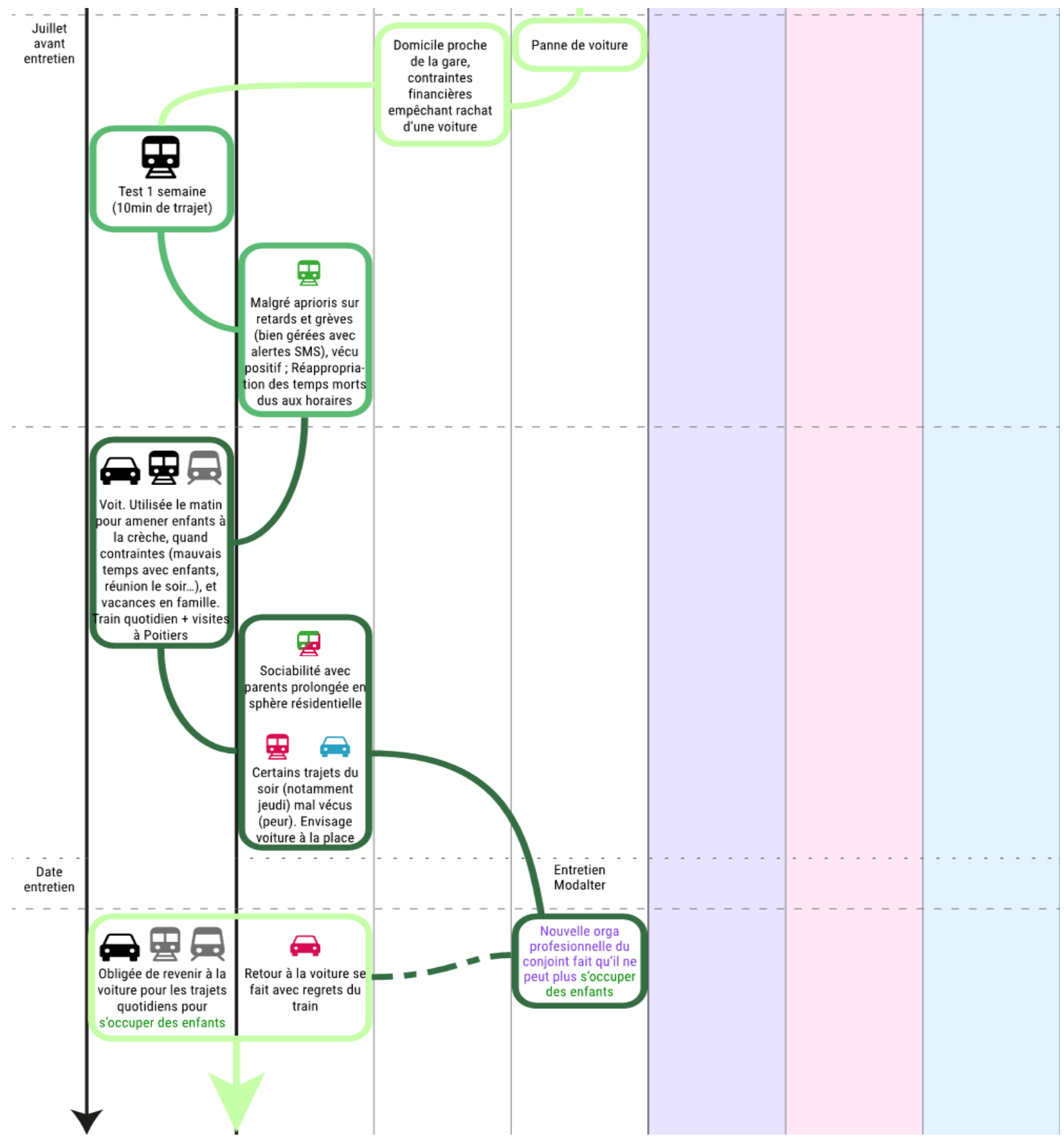

Penser les effets de socialisation à l'échelle du ménage

Enfin, on identifie un autre intérêt de la représentation graphique de la trajectoire mobilitaire : pouvoir visualiser, avec le code couleur violet pour le conjoint et vert pour les enfants, les effets des pratiques, compétences et représentations de la mobilité des autres membres du ménage sur l'individu (et réciproquement). On peut ainsi illustrer comment les trajectoires des 
individus sont aussi influencées par les pratiques des autres membres du ménage. Ces effets de socialisation croisés sont particulièrement visibles dans la trajectoire de Mme Da Silva, non seulement avec l'effet du changement d'horaires professionnels de son conjoint sur son retour à la voiture, mais également avec le choix du mode pour les trajets de loisirs du weekend qui fait l'objet d'intenses négociations avec son conjoint, habitué depuis très longtemps à se déplacer uniquement en voiture.

L'analyse des trajectoires de nos enquêtés montre ainsi comment leur changement de comportement se traduit par des évolutions des pratiques mobilitaires et de la motilité des autres membres de la famille. Expérimenter un nouveau mode induit par exemple le développement de connaissances sur le fonctionnement d'un mode et de compétences qui peuvent ensuite se diffuser chez d'autres membres de la famille, et induire des changements de comportement indirects, comme le montre l'exemple de Mme Pia ${ }^{4}$. Celle-ci, après avoir utilisé pendant des années sa voiture, se déplace en train pour ses déplacements pendulaires. Elle a donc développé des connaissances et une maîtrise de ce mode. Ces nouvelles connaissances et compétences se sont diffusées au reste de la famille, en particulier à son mari: il a développé une connaissance indirecte du mode (ses horaires, son mode de fonctionnement, ses tarifs, etc.) et pu établir une comparaison avec la voiture. Ainsi, c'est lui qui a proposé de prendre le train pour leurs déplacements vers Tours le samedi (alors qu'ils n'y avaient jamais pensé avant le report modal de Mme Pia), c'est lui qui a fait remarquer qu'utiliser le train était plus intéressant financièrement que la voiture (compte tenu de l'abonnement de Mme Pia, du coût du parking, etc.). Une autre enquêtée a quant à elle utilisé le TER et le bus pendant plusieurs années lorsqu'elle se rendait à Tours (et en avait donc une bonne maîtrise). Elle avait gardé de ces expériences un souvenir relativement négatif (puisqu'elle associait ces pratiques à la dépendance qu'elle ressentait); lors de nos différents entretiens, nous avons plusieurs fois évoqué le train et le bus, ses expériences avec ces modes, leurs horaires, tarifs, etc. Alors qu'elle ne l'avait pas emprunté depuis des années, elle a, suite à nos entretiens, conseillé à sa fille qui souhaitait aller à Tours, de prendre le TER. On voit ici comment les connaissances acquises par un membre de la famille peuvent se diffuser à d'autres, même des années après.

\section{Conclusion}

La notion de trajectoire mobilitaire et sa représentation graphique nous semblent porteuses de fortes potentialités, en premier lieu dans une optique scientifique de meilleure compréhension des processus d'expérimentations modales à différentes échelles temporelles. Ainsi, cette notion permet d'appréhender de manière plus complexe les pratiques de mobilité des individus, comme une succession ou une hybridation de pratiques modales, ce qui permet par exemple d'échapper à la présentation habituelle et stéréotypée de ménages ruraux et périurbains attachés frénétiquement à la voiture. L'application de la notion à d'autres contextes territoriaux permettrait alors de mieux cerner l'influence propre du territoire dans les trajectoires des individus. Quant à la représentation graphique, outre ses vertus d'aide à l'analyse et de support à l'argumentation du chercheur, un autre intérêt, pas encore exploré, serait de servir de support de réactivation auprès des enquêtés, afin de valider ou complexifier avec eux les résultats initiaux de la recherche, sur la base d'une démarche réflexive visant à développer l'auto-compréhension des processus de changement. Il est ainsi possible

\footnotetext{
4 La représentation graphique de sa trajectoire est disponible sur le site du Forum Vies Mobiles, à l'adresse suivante :....
} 
d'imaginer que l'enquêté ait une autre version du «fil interprétatif » que celle du chercheur et/ou y ajoute des éléments non évoqués lors du premier entretien.

La notion et la représentation graphique des trajectoires mobilitaires revêtent également un intérêt dans une perspective opérationnelle, en constituant un outil d'aide à la réflexion pour l'élaboration des politiques de mobilité. Elle permet, nous l'avons vu, de mieux comprendre l'impact des différentes familles de facteurs (vécu, compétences, perturbations extérieures, etc.) sur les choix modaux des individus. Cette meilleure compréhension peut servir à éclairer les politiques de mobilité, par exemple au sujet des tarifications dans les transports, ou du rôle du confort et du vécu du déplacement dans l'adoption d'un mode. Elle semble également intéressante dans le cadre des opérations de marketing individualisé, qui cherchent à encourager l'évolution des pratiques de mobilité individuelles vers des pratiques plus " durables » à travers des mesures de communication et sensibilisation, de formation, d'expérimentation (Huyghe, 2015 ; Rocci, 2015). Dans cette optique, la représentation graphique de la trajectoire de l'individu permettrait aux professionnels de disposer rapidement d'une vision d'ensemble de l'histoire mobilitaire de l'individu (valeurs, compétences acquises, expériences positives ou négatives vécues avec les différents modes, etc.) et de déterminer plus facilement les formations et le niveau d'accompagnement au changement les plus pertinents à proposer à l'individu.

Afin d'explorer plus avant les potentialités de la notion de trajectoire mobilitaire, la représentation graphique que nous proposons peut néanmoins être étoffée.

Pour donner sa pleine mesure, cet outil suppose tout d'abord d'être nourri par des méthodes de recueil de données adaptées : outre les récits de vie, d'autres méthodes d'enquête sont particulièrement adaptées à l'élaboration de trajectoires mobilitaires, à l'image des life course calendars (Schoenduwe et alii, 2015). Il serait également possible de s'inspirer de différentes enquêtes biographiques de l'INED, retraçant les trajectoires familiales, professionnelles et résidentielles d'individus, en y incluant un volet sur les pratiques de mobilité. Ces méthodes reposent néanmoins essentiellement sur la mémoire des enquêtés, ce qui questionne l'exactitude des éléments recensés, en particulier sur les expérimentations courtes ou n'ayant pas abouti à des pratiques pérennes, les tâtonnements, voire les projets de changement jamais mis en œuvre. Les enquêtes longitudinales menées auprès de panels interrogés plusieurs fois au cours du temps (Mercklé, 2015), qui donnent la possibilité de « reconstituer les trajectoires individuelles et les logiques de leurs transformations [...] d'une façon que les séries transversales ne permettent que rarement », constituent une alternative intéressante. Cette méthode, déjà mise en œuvre par Rocci (2007) dans ses recherches sur les changements modaux, permet d'éviter les biais liés aux défaillances mémorielles, mais, dans le cas d'un échantillon plus important, est coûteuse et pose des problèmes d'attrition des enquêtés au cours du temps.

Par ailleurs, notre représentation est centrée en l'état sur les choix modaux. L'intégration d'autres dimensions du système de mobilité, comme la forme ou l'échelle spatiale des territoires pratiqués, permettrait sans doute de montrer l'opérationnalité de la notion de trajectoire mobilitaire non seulement dans l'optique de diminuer l'usage individuel de la voiture mais également pour faire face aux inégalités d'accès à l'emploi ou aux services. Le chantier reste ouvert. 
Laurent Cailly est maître de conférences en géographie (Université de Tours, UMR 7324 CNRS-CITERES). Ses travaux portent sur l'évolution des modes d'habiter, des stratégies résidentielles et des mobilités quotidiennes dans les espaces périurbains français. Il a codirigé plusieurs ouvrages dont France. Une géographie urbaine, Armand Colin, 2010 ; Habiter les territoires périurbains, PUR, 2012 ; Les Espaces du logement, L'Harmattan, 2016. laurent.cailly@univ-tours.fr

Marie Huyghe est docteure en Aménagement de l'espace et Urbanisme et consultante indépendante. Ses thèmes de recherche concernent les modes d'habiter ruraux, en particulier les pratiques mobilitaires et leur potentiel d'évolution, qu'elle interroge à travers une approche comportementale. Avec les collectivités, elle travaille à l'élaboration et la mise en ouvre de politiques de mobilité durable.

huyghe@univ-tours.fr

Nicolas Oppenchaim est maître de conférences en sociologie (Université de Tours, UMR

7324 CNRS-CITERES). Il a travaillé sur les enfants sans-logement et sur les mobilités urbaines d'adolescents vivant dans des quartiers ségrégués. Il a publié en 2016 Adolescents de cité. L'épreuve de la mobilité, et en 2019 le Manuel de sociologie des mobilités géographiques (avec Leslie Belton-Chevallier et Stephanie Vincent-Geslin). nicolas.oppenchaim@univ-tours.fr

\section{Bibliographie}

Aguiléra A., Cacciari J., 2020, Living with fewer cars: review and challenges on household demotorization, Transport Reviews, DOI: 10.1080/01441647.2020.1772405

Authier J.-Y. (dir.), 2010, État des lieux sur les trajectoires résidentielles, PUCA. [En ligne] (consulté le 26 janvier 2020) Disponible à l'adresse : http://www.urbanismepuca.gouv.fr/IMG/pdf/rapport_trajectoires_resid_2010.pdf

Baslington H., 2008, Travel Socialization: A Social Theory of Travel Mode Behavior, International Journal of Sustainable Transportation, vol. 2, $\mathrm{n}^{\circ}$ 2, p. 91-114.

Cacciari J., 2020, Les Mobility Biographies Research et les Travel Socialization Studies sous le regard de la sociologie de la socialisation : vers l'examen de la production sociale des choix de mobilité spatiale, Espaces-temps.net [en ligne].

Cailly L., 2007, Capital spatial, stratégies résidentielles et processus d'individualisation, Annales de géographie, vol. 654, n², p.169-187.

Cailly L., 2008, Existe-t-il un mode d'habiter spécifiquement périurbain ?, EspacesTemps.net. [En ligne] (consulté le 26 janvier 2020) Disponible à l'adresse : https://www.espacestemps.net/articles/mode-habiter-periurbain/

Deleuil J., Barbey E., Sintès A., 2017, Le dévoiturage ou la ville sans (sa) voiture : mobilités plurielles, services numériques et vie de quartier, Flux, 2017/2 ( $\left.{ }^{\circ} 108\right)$, p. 80-87. DOI : 10.3917/flux1.108.0080

Dodier R., 2015, Innover dans la mobilité : une nécessité pour rendre les campagnes périurbaines habitables ?, Géocarrefour, n 90, vol. 4, p. 297-306. 
Dureau F., Imbert C., 2014, L'approche biographique des mobilités résidentielles, in : C. Imbert, Dubucs H., Dureau F., Giroud M. (dir.), D'une métropole à l'autre. Pratiques urbaines et circulations dans l'espace européen, Paris : Armand Colin, p. 33-79.

Greene M., Rau H., 2016, Moving across the life course: A biographic approach to researching dynamics of everyday mobility practices, Journal of Consumer Culture, vol. 18, p 60-82.

Grossetti M., 2006, L’imprévisibilité dans les parcours sociaux, Cahiers internationaux de sociologie, $\mathrm{n}^{\circ} 120$, p. 5-28.

Huyghe M., 2015, Habiter les territoires ruraux-Comprendre les dynamiques spatiales et sociales à l'oeuvre, évaluer les perspectives d'évolution des pratiques de mobilité des ménages, Thèse de doctorat en Aménagement de l'espace et Urbanisme sous la direction de A. Aguilera et S. Thibault, Université de Tours.

Javeau C., 2006, Routines quotidiennes et moments fatidiques, Cahiers internationaux de sociologie, $\mathrm{n}^{\circ} 121$, p. 227-238.

Kaufmann V., Tabaka K., Louvet N., Guidez J.-M., 2010, Et si les Français n'avaient plus seulement une voiture dans la tête? Évolution de l'image des modes de transports, Lyon : Éditions du CERTU.

Kaufmann V., 2014, Retour sur la ville : motilité et transformations urbaines, Lausanne : Presses Polytechniques et universitaires romandes.

Lanzendorf M., 2010, Key Events and Their Effect on Mobility Biographies: The Case of Childbirth, International Journal of Sustainable Transportation, vol.4, n5, p. 272-292.

Lesnard L., Saint Pol T., 2006, Introduction aux méthodes d'appariement optimal, Bulletin de méthodologie sociologique, $\mathrm{n}^{\circ} 90$, p. 5-25.

Lévy J., 2003, Périurbain, le choix n'est pas neutre, Pouvoirs locaux, n 56, p. 35-42.

Rau H., Manton R., 2016, Life events and mobility milestones: Advances in mobility biography theory and research, Journal of Transport Geography, ${ }^{\circ}$ 52, p.51-60.

Mattioli G., 2019, La socialisation aux/par la mobilité à longue distance : l'approche par les biographies de mobilité, communication à la journée d'études Socialisation aux pratiques de mobilité / socialisation par les pratiques de mobilité, Université Paris-Est, Champs-sur-Marne, 4 avril 2019.

Mercklé P., 2015, Analyse longitudinale, in: Paugam S. (dir), Les 100 mots de la sociologie, Paris : PUF, p. 24-26.

Müggenburg H., Busch-Geertsema A., Lanzendorf M., 2015, Mobility biographies: A review of achievements and challenges of the mobility biographies approach and a framework for further research, Journal of Transport Geography, n 46, p. 151-163.

Oppenchaim N., 2016, Adolescents de cité. L'épreuve de la mobilité, Tours : Presses Universitaires François Rabelais. 
Oppenchaim N., Fouquet J.-P., Pourtau B., 2017. Les changements de modes de déplacement dans le périurbain, EspacesTemps.net. [En ligne] (consulté le 26 janvier 2020) Disponible à l'adresse : https://hal.archives-ouvertes.fr/hal-01705478/document

Prochaska J. O., Di Clemente C., 1982, Trans-theoretical therapy - toward a more integrative model of change, Psychotherapy: Theory, Research, and Practice, $\mathrm{n}^{\circ} 19, \mathrm{p} .276-288$.

Rocci A., 2007, De l'automobilité à la multimodalité, Thèse de doctorat en sociologie sous la direction de D. Desjeux, Université Paris 5.

Rocci A., 2015, Comment rompre avec l'habitude ? Les programmes d'accompagnement au changement de comportements de mobilité, Espace Populations sociétés, n ${ }^{\circ} 1-2$. [En ligne] (consulté le 26 janvier 2020) Disponible à l'adresse : https://journals.openedition.org/eps/6027

Schoenduwe R., Mueller M. G., Peters A., Lanzendorf M., 2015, Analysing mobility biographies with the life course calendar: a retrospective survey methodology for longitudinal data collection, Journal of Transport Geography, n ${ }^{\circ} 2$, p. 98-109.

Vincent-Geslin S., 2010, Altermobilités, mode d'emploi, Déterminants et usages de mobilités alternatives au tout voiture, Lyon : Éditions du CERTU. 